\title{
Low dose anti-thymocyte globulin with low dose posttransplant cyclophosphamide (low dose ATG/PTCy) can reduce the risk of graft- versus-host disease as compared with standard-dose anti-thymocyte globulin in haploidentical peripheral hematopoietic stem cell transplantation combined with unrelated cord blood
}

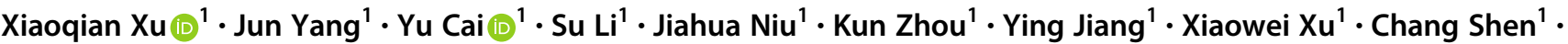

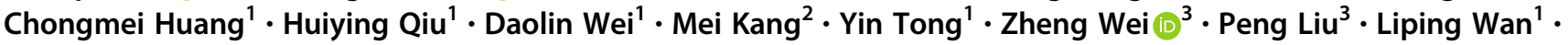 \\ Xianmin Song ${ }^{1}{ }^{1}$
}

Received: 18 October 2019 / Revised: 7 August 2020 / Accepted: 25 August 2020 / Published online: 1 September 2020

(C) The Author(s) 2020. This article is published with open access

\section{To the Editor:}

For successful transplantation using a haploidentical donor, it requires effective depletion of $\mathrm{T}$ cells to reduce the risk of graft-versus-host disease (GvHD) and prevent serious GvHD. Contemporary practice of haploidentical hematopoietic stem cell transplantation (Haplo-HSCT) frequently adopts in vivo T-cell depletion strategies: a monoclonal antibody against $\mathrm{T}$ cells using antihuman thymocyte immunoglobulin (ATG) $[1,2]$ or posttransplant cyclophosphamide (PTCy) based regimen [3, 4].

The ATG-based regimen was one of the most commonly used GvHD prophylaxis for Haplo-HSCT in China but remaining an issue of higher risk of acute GvHD (aGvHD)

These authors contributed equally: Xiaoqian Xu, Jun Yang

Supplementary information The online version of this article (https:// doi.org/10.1038/s41409-020-01047-2) contains supplementary material, which is available to authorized users.

Liping Wan

lipingwan@sjtu.edu.cn

Xianmin Song

shongxm@sjtu.edu.cn

1 Department of Hematology, Shanghai General Hospital, Shanghai Jiao Tong University, No. 100 Haining Road, 200080

Shanghai, China

2 Clinical Research Center, Shanghai General Hospital, Shanghai Jiao Tong University, Shanghai, China

3 Department of Hematology, Zhongshan Hospital, Fudan University, Shanghai, China up to $40 \%$ incidence $[1,2]$ as well as cytomegalovirus (CMV) and Epstein-Barr virus (EBV) infection. PTCybased regimen had outstanding outcomes of GvHD prevention in Haplo-HSCT with bone marrow grafts with $21-34 \%$ of incidences of grades II-IV aGvHD. However, by substituting BM graft with peripheral blood stem cell (PBSC) grafts, the incidence of grades II-IV aGvHD increased in Haplo-HSCT even after the use of PTCy-based regimens for GvHD prophylaxis [5, 6]. Accordingly, there is room for further improvement of GvHD reduction when the haploidentical transplant was performed using a PBSC graft. Thus there is a practical demand for more powerful GvHD prophylaxis for haploidentical peripheral blood stem cell transplantation (Haplo-PBSCT) beyond ATG-based or PTCy-based regimens.

Recently, we have applied a novel regimen of combining low dose ATG $(5 \mathrm{mg} / \mathrm{kg}$ ) with low dose PTCy (one dose of $50 \mathrm{mg} / \mathrm{kg}$ ) (low dose ATG/PTCy) for prevention of GvHD in Haplo-HSCT with PBSC grafts combined with unrelated single cord blood (UCB) in our center [7]. The novel GvHD prophylaxis regimen showed promising activity with grades II-IV aGvHD of $19.4 \%$, which was significantly lower than that with other regimens including standard PTCy regimen (2 days Cy $100 \mathrm{mg} / \mathrm{kg}$ ) [4-7]. Some studies [8-11] reported that UCB in combination with $\mathrm{CD} 34^{+}$selected PBSCs from a related mismatched donor were transplanted into recipients with fast engraftment, low incidences of GvHD, and promising long-term results. According to the results in these studies, an UCB was co-transfused with PBSCs in our study, while an UCB as the third party cells might obscure the effects of the novel regimen in the prophylaxis of GvHD. Thus to confirm the effects of the novel regimen on 
GvHD prophylaxis, two cohorts of patients received the same grafts (PBSCs plus a UCB) with standard-dose ATG or low doseATG/PTCy-based regimens were comparatively analyzed.

From January 2014 to June 2018, 36 patients with myeloid malignancies in standard-dose ATG group were enrolled in this trial, while 31 cases in low dose ATG/PTCy group were from June 2017. Eight cases in our previous study [7] were excluded from the current study because of five cases with ALL and three myeloid malignancy cases with loss to follow-up. Informed consent was obtained from all subjects, and the study protocol was approved by the local Ethical Review Board of the Shanghai General Hospital, Shanghai, China. The patients younger than 55 years old received myeloablative conditioning (MAC), while those equal to or older than 55 years of age received reduced-intensity conditioning (RIC). All patients received BFA conditioning regimens consisted of Fludarabine and Cytarabine with different doses of Busulfan $(3.2 \mathrm{mg} / \mathrm{kg} / \mathrm{day}$ for 3-4 days in cases of MAC, while $3.2 \mathrm{mg} / \mathrm{kg} /$ day for 2 days in RIC) [7, 12].

In the group received low dose ATG/PTCy, ATG was given at $2.5 \mathrm{mg} / \mathrm{kg} /$ day for 2 days $(\mathrm{d}-2, \mathrm{~d}-1$; a total of $5 \mathrm{mg} / \mathrm{kg}$ ) and a dose of cyclophosphamide $(50 \mathrm{mg} / \mathrm{kg} /$ day $)$ was given for 1 day on $d+3$. Cyclosporine A (CSA) was given from $\mathrm{d}+4$. Mycophenolate mofetil (MMF) was administered orally at a dose of $1 \mathrm{~g}$ three times a day from $\mathrm{d}+1$ to $\mathrm{d}+34$ if aGvHD does not occur [7]. In the group received a standard-dose ATG-based regimen, ATG was given at a dose of $2.5 \mathrm{mg} / \mathrm{kg} / \mathrm{d}$ from $\mathrm{d}-4$ to $\mathrm{d}-1$, a total of $10 \mathrm{mg} / \mathrm{kg}$. CSA was given from $\mathrm{d}-5$. MMF was administered at a dose of $1 \mathrm{~g}$ three times a day from $\mathrm{d}+1$ to $\mathrm{d}+$ 30. Methotrexate (MTX) was added at a dose of $10 \mathrm{mg} / \mathrm{m}^{2}$ on $\mathrm{d}+1$, and $5 \mathrm{mg} / \mathrm{m}^{2}$ on days +3 and +6 .

A total of 31 cases received a low dose ATG/PTCy, while 36 cases received a standard-dose ATG. No significant differences were observed in age, gender, HCT-CI, conditioning regimen, donor type, infused cell numbers of PBSCs, and UCB grafts between the two groups (Supplementary Table 1). The percentage of AML-CR1 patients in low dose ATG/PTCy group was significantly higher than that in standard-dose ATG group (32.3 vs. $8.3 \%, P=$ $0.014)$. While the percentage of MDS-MLD patients in low dose ATG/PTCy group was significantly lower than that in standard-dose ATG group (0.0 vs. $19.4 \%, P=0.028)$. In addition, there was no significant difference $(P=0.422)$ between two groups in revised-disease risk index (R-DRI). As expected, the follow-up duration was longer in standard ATG group (median 23 months) than in low dose ATG/ PTCy group (median 12.5 months; $P=0.004$ ) because the use of low dose ATG/PTCy had been recently introduced while the standard-dose ATG regimen was a standard GvHD prophylaxis for longer period of time in our center.
With respect to engraftment, no differences were noted between the two groups in neutrophil $(P=0.712)$ and platelet engraftment $(P=0.312)$. The primary graft failure rate was similar: $3.2 \%(n=1 / 31)$ in the low dose ATG/PTCy group and 2.8\% $(n=1 / 36)$ in the standard-dose ATG group $(P=1.000)$. Secondary graft failures (SGFs) were observed in two cases without donor chimerism (graft rejection) in low dose ATG/PTCy group, which occurred at $+24 \mathrm{~d}$ and $+80 \mathrm{~d}$ after transplantation in the context of infection. The two cases were diagnosed with AML and received MAC regimens. Although no patient developed SGF in the standard-dose ATG group, there was no difference in the risk of SGF $(P=0.272)$ between two groups.

In terms of GvHD risk, the risk of aGvHD was significantly lower in the low dose ATG/PTCy group: the incidence of grades II-IV aGvHD was $17.0 \%$ in low dose ATG/PTCy $(n=5)$ within 180 days vs. $40.2 \%$ in standard ATG group $(n=14 ; P=0.042$; Table 1 and Supplementary Fig. 1).There was no significant differences in the incidence of chronic GVHD between the two groups. While, the rate of moderate to severe cGvHD was significantly lower as $11.2 \%$ at 1-year in low dose ATG/PTCy group as compared to $40.1 \%$ in the standard-dose ATG group $(P=0.029)$. The low dose ATG/PTCy group seemed to have a reduced trend of CMV and EBV infection compared to the standard-dose ATG group (Table 1).

However, long-term transplant outcomes did not show any significant differences between the two groups concerning overall survival $(P=0.255)$ and leukemia-free survival $(P=$ 0.400; Table 1 and Supplementary Fig. 2). The non-relapse mortality rate was lower in the low dose ATG/PTCy group as $12.9 \%$ at 1 year than in standard-dose ATG group (36.2\%; $P=0.038$ ), while the incidence of relapse was higher in the low dose ATG/PTCy group as $22.9 \%$ at 1 year than in the standard-dose ATG group $(8.7 \% ; P=0.042)$.

The key finding of the present study is that the risk of grades II-IV aGvHD and moderate to severe cGvHD were significantly lower in the low dose ATG/PTCy group than that in the standard ATG group, suggesting that low dose ATG/PTCy regimen is very promising for prevention of GvHD in Haplo-PBSCT which could potentially improve GvHD-free, relapse-free survival after Haplo-HSCT. The rationale of using a combined regimen of ATG and PTCy is that administration of low dose ATG could deplete early active T-lymphocytes while an infusion of PTCy on day +3 could eradicate rapidly proliferating $\mathrm{T}$ cells after antigen exposure [4], which could exert a synergistic activity to reduce the risk of GvHD because they have different action mechanisms on T-lymphocyte depletion.

Of note, the low dose ATG/PTCy regimen can reduce the risk of NRM significantly: $12.9 \%$ of NRM in the low dose ATG/PTCy group vs. $36.2 \%$ of NRM in the standard ATG group. It can be explained by the extremely lower rate of 
Table 1 Clinical outcomes of patients in the two groups.

\begin{tabular}{|c|c|c|c|}
\hline & $\begin{array}{l}\text { Low dose ATG/ } \\
\text { PTCy regimen } \\
(n=31)\end{array}$ & $\begin{array}{l}\text { Standard-dose } \\
\text { ATG regimen } \\
(n=36)\end{array}$ & $P$ \\
\hline $\begin{array}{l}\text { CI of grades I-IV } \\
\text { aGvHD at } 180 \text { days }\end{array}$ & $49.7 \%$ & $79.3 \%$ & 0.065 \\
\hline $\begin{array}{l}\text { CI of grades II-IV } \\
\mathrm{aGvHD} \text { at } 180 \text { days }\end{array}$ & $17.0 \%$ & $40.2 \%$ & 0.042 \\
\hline $\begin{array}{l}\text { CI of grades III-IV } \\
\text { aGvHD at } 180 \text { days }\end{array}$ & $3.2 \%$ & $23.1 \%$ & 0.025 \\
\hline $\mathrm{CI}$ of $\mathrm{cGVHD}$ at 1 year & $42.7 \%$ & $57.7 \%$ & 0.190 \\
\hline $\begin{array}{l}\text { CI of moderate to } \\
\text { severe cGVHD at } 1 \text { year }\end{array}$ & $11.2 \%$ & $40.1 \%$ & 0.029 \\
\hline \multicolumn{4}{|l|}{ Viral infection } \\
\hline CMV viremia & $13(41.9 \%)$ & $23(63.8 \%)$ & 0.072 \\
\hline CMV disease & $5(16.1 \%)$ & $5(13.9 \%)$ & 1.000 \\
\hline EB viremia & $14(45.2 \%)$ & $24(66.7 \%)$ & 0.076 \\
\hline PTLD EBV related & $0(0.0 \%)$ & $1(2.8 \%)$ & 1.000 \\
\hline CIR at 1 year & $22.9 \%$ & $8.7 \%$ & 0.042 \\
\hline NRM at 1 year & $12.9 \%$ & $36.2 \%$ & 0.038 \\
\hline \multicolumn{4}{|l|}{ Causes of NRM } \\
\hline GvHD & $0(0 \%)$ & $7(23.3 \%)$ & 0.028 \\
\hline Infection & $0(0 \%)$ & $5(13.9 \%)$ & 0.091 \\
\hline MOF & $1(3.2 \%)$ & $1(2.8 \%)$ & 1.000 \\
\hline $\begin{array}{l}\text { Graft failure/ } \\
\text { dysfunction }\end{array}$ & $3(9.7 \%)$ & $1(2.8 \%)$ & 0.502 \\
\hline $\begin{array}{l}\text { Early mortality (Within } \\
3 \text { months post } \\
\text { transplant) }\end{array}$ & $3(9.7 \%)$ & $11(30.6 \%)$ & 0.036 \\
\hline OS (1 year) & $74.9 \%$ & $59.4 \%$ & 0.255 \\
\hline LFS (1 year) & $64.2 \%$ & $55.0 \%$ & 0.400 \\
\hline
\end{tabular}

$C M V$ cytomegalovirus, PTLD posttransplant lymphoproliferative disorder, $C I R$ cumulative incidence of relapse, $C I$ cumulative incidence, NRM non-relapse mortality, MOF multiple organ failure, $O S$ overall survival, LFS leukemia-free survival.

GvHD related death in the low dose ATG/PTCy group compared to that in the standard-dose ATG group $(P=$ 0.028). The incidences of infection were not significantly different between the two groups. However, the infectionrelated mortality rate was substantially higher in the standard ATG group compared to that in the low dose ATG/ PTCy group (9/36 vs. $1 / 31, P=0.032$ ). Our personal experience is that the infectious complications in the cases received standard-dose ATG were much more challenging to control than those received low dose ATG/PTCy. It could be plausible that standard-dose ATG is more immunosuppressive, especially for T-cell immunity than low dose ATG/PTCy regimen, thus resulting in prolonged immunosuppression.

The risk of relapse was higher in the low dose ATG/ PTCy group compared to the standard ATG group (22.8 vs.
$8.7 \%$ ). Of note, more patients died at earlier time (within 3 months) in the standard-dose ATG group (30.6\%) compared to the low dose ATG/PTCy group $(9.7 \%, P=0.036)$, which might be the major reason for a lower relapse rate in the standard-dose ATG group because the median relapse time was 7 months in overall patients. The relapse incidences in the present study were higher than that in Ruggeri's et al. [13] and Salvatore's et al. [14] studies. However, it requires cautious interpretation because of different disease characteristics among the studies. Our study included more patients with advanced disease close to $60 \%$ (19/31 and 21/36, respectively), while higher numbers of patients in remission were included in the Ruggeri's et al. [13] (100\% in CR1) and Salvatore's et al. [14] studies (69\% in CR1).

Although it did not reach statistical significances, there were strong trends of reduced incidences of CMV and EBV infection in the low dose ATG/PTCy group in comparison to the standard-dose ATG group. The incidence of CMV viremia in the low dose ATG/PTCy group was similar to that with standard PTCy regimen in a range of 38-50\% $[4,15,16]$ of CMV viremia, while it was much higher when received ATG-based regimens with the incidence of CMV viremia over $60 \%$ [2].

In summary, the low dose ATG/ PTCy was feasible and could effectively reduce the risk of GvHD and lower the NRM in comparison to the standard-dose ATG. A prospective randomized trial would be required to reach a definite conclusion on the superior efficacy of low dose ATG/PTCy regimen in Haplo-PBSCT.

Acknowledgements We thank the nursing staff for providing excellent care for our patients. This study was funded by Clinical Research Plan of SHDC (Cultivation project) (SHDC12018X09), Clinical Research Innovation Plan of Shanghai General Hospital (CTCCR-2018BP03), Science and technology commision of shanghai municipality (Medical Guidance Project) (18411968400), Shanghai Municipal Health and Family Planning Commission Clinical Research Special General Project (201840043), National Key R\&D Program of China (2019YFA011100 0), 3-year development project from Shanghai Shenkang Hospital Development Center (16CR1010A), National Natural Science Foundation of China (81570148).

Author contributions XQX, JY, XMS, and LPW designed the research, collected the data, and wrote the paper. MK analyzed the data. YC, SL, JHN, KZ, YJ, XWX, CS, CMH, HYQ, DLW, YT, ZW, and PL assisted in data collection and paper preparation. All the authors approved the final draft of the paper.

\section{Compliance with ethical standards}

Conflict of interest The authors declare that they have no conflict of interest.

Publisher's note Springer Nature remains neutral with regard to jurisdictional claims in published maps and institutional affiliations. 
Open Access This article is licensed under a Creative Commons Attribution 4.0 International License, which permits use, sharing, adaptation, distribution and reproduction in any medium or format, as long as you give appropriate credit to the original author(s) and the source, provide a link to the Creative Commons license, and indicate if changes were made. The images or other third party material in this article are included in the article's Creative Commons license, unless indicated otherwise in a credit line to the material. If material is not included in the article's Creative Commons license and your intended use is not permitted by statutory regulation or exceeds the permitted use, you will need to obtain permission directly from the copyright holder. To view a copy of this license, visit http://creativecommons. org/licenses/by/4.0/.

\section{References}

1. Huang XJ, Liu DH, Liu KY, Xu LP, Chen H, Han W, et al. Haploidentical hematopoietic stem cell transplantation without in vitro T-cell depletion for the treatment of hematological malignancies. Bone Marrow Transplant. 2006;38:291-7.

2. Wang Y, Liu DH, Liu KY, Xu LP, Zhang XH, Han W, et al. Long-term follow up of haploidentical hematopoietic stem cell transplantation without in vitro $\mathrm{T}$ cell depletion for the treatment of leukemia: nine years of experience at a single center. Cancer. 2013;119:978-85.

3. Luznik L, Jalla S, Engstrom LW, Iannone R, Fuchs EJ. Durable engraftment of major histocompatibility complex-incompatible cells after nonmyeloablative conditioning with fludarabine, lowdose total body irradiation, and posttransplantation cyclophosphamide. Blood. 2001;98:3456-64.

4. Luznik L, O'Donnell PV, Symons HJ, Chen AR, Leffell MS, Zahurak M, et al. HLA-haploidentical bonemarrow transplantation for hematologic malignancies using nonmyeloablative conditioning and high-dose, posttransplantation cyclophosphamide. Biol Blood Marrow Transplant. 2008;14:641-50.

5. Bashey A, Zhang MJ, McCurdy SR, St Martin A, Argall T, Anasetti $\mathrm{C}$, et al. Mobilized peripheral blood stem cells versus unstimulated bone marrow as a graft source for T-cell-replete haploidentical donor transplantation using post-transplant cyclophosphamide. J Clin Oncol. 2017;35:3002-9.

6. Yu X, Liu L, Xie Z, Dong C, Zhao L, Zhang J, et al. Bone marrow versus peripheral blood as a graft source for haploidentical donor transplantation in adults using post-transplant cyclophosphamideA systematic review and meta-analysis. Crit Rev Oncol Hematol. 2019;133:120-8.

7. Yang J, Jiang J, Cai Y, Li S, Wan L, Zhu J, et al. Low-dose antithymocyte globulin plus low-dose posttransplant cyclophosphamide as graft-versus-host disease prophylaxis in haploidentical peripheral blood stem cell transplantation combined with unrelated cord blood for patients with hematologic malignancies: a prospective, phase II study. Bone Marrow Transplant. 2018;54: 1049-57.

8. Magro E, Regidor C, Cabrera RSanjuán I, Forès R, Garcia-Marco $\mathrm{JA}$, et al. Early hematopoietic recovery after single unit unrelated cord blood transplantation in adults supported by co-infusion of mobilized stem cells from a third party donor. Haematologica. 2006;91:640-8.

9. Bautista G, Cabrera JR, Regidor C, Forés R, García-Marco JA, Ojeda E, et al. Cord blood transplants supported by co-infusion of mobilized hematopoietic stem cells from a third-party donor. Bone Marrow Transplant. 2009;43:365-73.

10. Liu H, Rich ES, Godley L, Odenike O, Joseph L, Marino S, et al. Reduced-intensity conditioning with combined haploidentical and cord blood transplantation results in rapid engraftment, low GVHD, and durable remissions. Blood. 2011;118:6438-45.

11. Tsai SB, Rhodes J, Liu H, Shore T, Bishop M, Cushing MM, et al. Reduced-intensity allogeneic transplant for acute myeloid leukemia and myelodysplastic syndrome using combined CD34selected haploidentical graft and a single umbilical cord unit compared with matched unrelated donor stem cells in older adults. Biol Blood Marrow Transplant. 2018;24:997-1004.

12. Xu L, Chen H, Chen J, Han M, Huang H, Lai Y, et al. The consensus on indications, conditioning regimen, and donor selection of allogeneic hematopoietic cell transplantation for hematological diseases in China-recommendations from the Chinese Society of Hematology. J Hematol Oncol. 2018;11:33.

13. Ruggeri A, Labopin M, Bacigalupo A, Gülbas Z, Koc Y, Blaise $\mathrm{D}$, et al. Bone marrow versus mobilized peripheral blood stem cells in haploidentical transplants using posttransplantation cyclophosphamide. Cancer. 2018;124:1428-37.

14. Salvatore D, Labopin M, Ruggeri A, Battipaglia G, Ghavamzadeh A, Ciceri F, et al. Outcomes of hematopoietic stem cell transplantation from unmanipulated haploidentical versus matched sibling donor in patients with acute myeloid leukemia in first complete remission with intermediate or high-risk cytogenetics: a study from the Acute Leukemia Working Party of the European Society for Blood and Marrow Transplantation. Haematologica. 2018;103:1317-28.

15. Moiseev IS, Pirogova OV, Alyanski AL, Babenko EV, Gindina TL, Darskaya EI, et al. Graft-versus-host disease prophylaxis in unrelated peripheral blood stem cell transplantation with post-transplantation cyclophosphamide, tacrolimus, and mycophenolate mofetil. Biol Blood Marrow Transplant. 2016;22: 1037-42.

16. Raiola AM, Dominietto A, Ghiso A, Di Grazia C, Lamparelli T, Gualandi $\mathrm{F}$, et al. Unmanipulated haploidentical bone marrow transplantation and posttransplantation cyclophosphamide for hematologic malignancies after myeloablative conditioning. Biol Blood Marrow Transplant. 2013;19:117-22. 